\title{
The effect of crystallite size and stress condition on the equation of state of nanocrystalline $\mathrm{MgO}$
}

\author{
H. Marquardt, ${ }^{1, a)}$ S. Speziale, ${ }^{1}$ K. Marquardt, ${ }^{1}$ H. J. Reichmann, ${ }^{1}$ Z. Konôpková, ${ }^{2}$ \\ W. Morgenroth, ${ }^{3}$ and H.-P. Liermann ${ }^{2}$ \\ ${ }^{1}$ GFZ German Research Centre for Geosciences, Telegrafenberg, 14473 Potsdam, Germany \\ ${ }^{2}$ Deutsches Elektronensynchrotron DESY, Notkestr. 85, 22607 Hamburg, Germany \\ ${ }^{3}$ Goethe Universität Frankfurt, Altenhöferallee 1, 60438 Frankfurt am Main, Germany
}

(Received 25 July 2011; accepted 11 October 2011; published online 6 December 2011)

\begin{abstract}
We performed high-pressure synchrotron x-ray diffraction experiments on nanocrystalline (nc-) $\mathrm{MgO}$ compressed both under quasi-hydrostatic and non-hydrostatic conditions in a diamond-anvil cell. Data obtained under hydrostatic conditions show that $\mathrm{nc}-\mathrm{MgO}$ (average crystallite size of $20 \mathrm{~nm}$ ) is $8-9 \%$ more compressible than "bulk" MgO. Analysis of our results collected under non-hydrostatic conditions yields a bulk modulus that is about $27 \%$ larger than the one derived from the quasi-hydrostatic compression experiments. Thus, the apparent bulk modulus strongly depends on the experimental stress conditions. (C) 2011 American Institute of Physics.
\end{abstract}

[doi:10.1063/1.3662491]

\section{INTRODUCTION}

The properties of nanosized materials may differ from those of coarse-grained ("bulk") materials, because of their reduced crystallite size and large fraction of surfaces/interfaces, where the atomic arrangement is disordered as compared to the perfect crystal lattice (e.g., Refs. 1 and 2). The effect of very small crystallite sizes on the elastic properties of the crystalline cores is still not well understood, particularly at high pressure. Several studies used synchrotron x-ray diffraction on nanocrystalline powders at high pressures to derive both the isothermal bulk modulus $K_{T 0}$ and its pressure derivative at zero pressure $\left(\partial K_{T} / \partial P\right)_{T 0}$ (e.g., Refs. 3-9). However, most of these studies were conducted under strongly nonhydrostatic conditions to also reveal the interplay between crystallite size and compressive strength. ${ }^{10-13}$ Unfortunately, these experiments are not well suited to quantify any crystallite-size effect on the elastic compressibility ${ }^{11,14}$ and controversial results are reported in the literature. Some studies suggest that the compressibility of a nanocrystalline material significantly differs from its bulk counterpart, ${ }^{4,8,9}$ whereas others do not observe any change. ${ }^{5-7,14,15}$

In this study, we performed high-pressure synchrotron $\mathrm{x}$-ray diffraction experiments on nc-MgO powder compressed under quasi-hydrostatic conditions using either neon or argon (with different mechanical properties) as pressure-transmitting media or under strongly nonhydrostatic conditions (no pressure-transmitting medium). A Birch-Murnaghan equation of state ${ }^{16}$ was fitted to the pressure-dependent unit cell volumes to quantify the effects of crystallite size and non-hydrostaticity on the elastic compressibility of $\mathrm{MgO}$. In addition, the observed x-ray line broadening was used to derive information about the average crystallite size at high pressures.

${ }^{\text {a)} E l e c t r o n i c ~ m a i l: ~ h a m a @ g f z-p o t s d a m . d e . ~}$

\section{MATERIALS AND METHODS}

$\mathrm{MgO}$ powder with an average crystallite size of about $20 \mathrm{~nm}$ was used as starting material (American Elements, 99.9\% purity). Transmission electron microscopy (TEM) images are shown in Fig. 1. The TEM micrographs were acquired on a FEI Tecnai $\mathrm{F}^{20} \mathrm{X}$-Twin with a field emission gun operated at $200 \mathrm{kV}$. The presented images are energy filtered applying a $10-\mathrm{eV}$ window to the zero-loss peak using the Gatan Tridiem Imaging filter. The nc-MgO powder was loaded in symmetric piston cylinder diamond-anvil cells (DAC, Ref. 17), where either diamonds with $300 \mu \mathrm{m}$ or $400 \mu \mathrm{m}$-sized culets were employed. Rhenium was used as gasket material. Pressure was generated either by screws or by a membrane system. The applied pressure was determined by ruby fluorescence using existing calibrations for both quasi-hydrostatic and non-hydrostatic stress conditions. ${ }^{18,19}$ Four different experimental runs at varying degrees of nonhydrostaticity were performed to high pressures to test the effect of different stress conditions on the apparent equation of state. In the first experimental run, the nc-MgO powder was compressed without a pressure-transmitting medium. In runs two and three, argon and neon, respectively, were employed as a pressure-transmitting medium to create different quasi-hydrostatic sample environments. The fourth experiment was conducted on the recovered sample material from the non-hydrostatic experiment, which was re-loaded with neon as pressure-transmitting medium. In the two runs with neon, particular care was taken to ensure that the sample material was surrounded by the pressure-transmitting medium and no bridging between the diamond anvils occurred. These runs displayed the most hydrostatic conditions as indicated by the width of the ruby $\mathrm{R}_{1}$ fluorescence line.

High-pressure synchrotron $\mathrm{x}$-ray diffraction experiments were performed at DESY/PETRA III beamline P02.2 (Extreme Conditions Beamline) using x-rays with an energy of $42.7 \mathrm{keV}(0.29036 \AA$ wavelength $)$ and a focusing spot of $\sim 2.0(\mathrm{H}) \times 1.8(\mathrm{~V}) \mu \mathrm{m}^{2}$ (Ref. 20). The sample-detector 


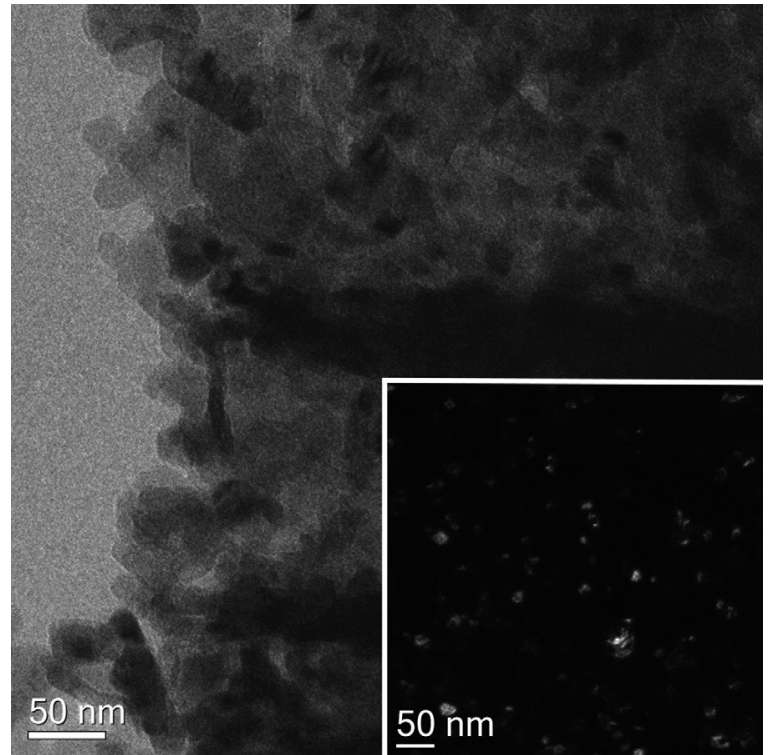

FIG. 1. TEM micrographs. Bright field image of a representative area of the powder sample. The inset in the lower right corner shows dark field image of a larger region using part of the [002] diffraction ring.

distance was calibrated using a $\mathrm{CeO}_{2}$ standard (NIST 674 a). Diffraction images were acquired on a Perkin Elmer XRD 1621 flat panel detector using the software qxrd (G. Jennings, Advanced Photon Source) with exposure times ranging from 30 to $90 \mathrm{~s}$. Up to nine diffraction lines were recorded at high-pressures. The program fit $2 \mathrm{~d}$ (Ref. 21) was used to convert the images to integrated diffraction patterns. An additional powder diffraction pattern was collected at ambient conditions using a STOE Stadi $\mathrm{P}$ diffractometer equipped with a $\mathrm{Cu}_{\mathrm{K} \alpha} \mathrm{X}$-ray tube. In the latter experiment, a silicon standard (NIST 640 c) was mixed together with the nc-MgO to directly calibrate the x-ray spectrum and detect slight changes in the unit cell parameter of nc- $\mathrm{MgO}$ as compared to "bulk" $\mathrm{MgO}$. Each peak in the integrated diffraction patterns was background-corrected and fit with a four parameter pseudo-Voigt function using the program PeakFit (version 4.11). The unit cell volumes at the corresponding pressures were calculated from the positions of the refined diffraction peaks. The reported $1 \sigma$-uncertainties are reflecting the differences in unit cell volumes calculated from the different $h k l$-reflections. After correcting for instrumental broadening, the $\mathrm{x}$-ray line widths were used to derive the average crystallite size by applying the identity given below, ${ }^{10,13,22}$ which represents an extension of the Scherrer equation $^{23,24}$ to high-pressure DAC experiments:

$$
\left(2 w_{h k l} \cos \theta_{h k l}\right)^{2}=(\lambda / d)^{2}+\left(4 p_{\max } / E_{h k l}\right)^{2} \sin ^{2} \theta_{h k l},
$$

where $2 w_{h k l}$ refers to the full width at half maximum (FWHM) of a specific reflection $h k l, \lambda$ is the x-ray wavelength, $d$ is the apparent average crystallite size and $E_{h k l}$ refers to the (high-pressure) Young's modulus along $[h k l] .{ }^{10}$ $2 p_{\text {max }}$ denotes the difference between the maximum and minimum stress applied to the crystallites. ${ }^{10,11,22}$ A previous high-pressure $\mathrm{X}$-ray diffraction study $^{13}$ suggests that $2 p_{\max }$ derived from Eq. (1) is a reliable measure of compressive strength $\sigma$. This conclusion was based on the good agreement between the compressive strength of gold derived by applying Eq. (1) and the results from hardness measurements. However, for the purpose of this study, $2 p_{\max }$ is not analyzed to extract information about the strength of nc- $\mathrm{MgO}$ at high pressures. The apparent average crystallite size was derived from the intercept in a $\left(2 w_{h k l} \cos \theta_{h k l}\right)^{2}$ versus $\left(\sin \theta_{h k l} / E_{h k l}\right)^{2}$ plot and multiplied with a shape factor of 0.9 , which has been derived for cubic materials, ${ }^{25,26}$ to determine the "true" average crystallite size. Elastic constants from a computational study ${ }^{27}$ were used to calculate Young's modulus at high pressure.

\section{RESULTS AND DISCUSSION}

The analysis of our x-ray diffraction pattern collected outside the DAC resulted in an apparent zero pressure unit cell volume of $74.90( \pm 0.03) \AA^{3}$ for nc-MgO. This observation is consistent with our measurements in the DAC before non-hydrostatic compression (run \#1), where we determined the unit cell volume to be 74.91 ( \pm 0.04$) \AA^{3}$ (Fig. 2). The increased unit cell volume as compared to "bulk" $\mathrm{MgO}$ $\left(74.71 \pm 0.01 \AA^{3}\right.$, Ref. 28$)$ indicates that the nc-MgO is in a state of lattice expansion, probably due to excess volume in the surface/interface regions (e.g., Ref. 29). Such a lattice expansion has previously been reported for different nanomaterials. ${ }^{30,31}$ We extracted an average crystallite size of $19.7( \pm 0.2) \mathrm{nm}$ from analysis of the x-ray linewidth broadening of the starting material (Fig. 3). The reported uncertainty reflects the statistical $1 \sigma$-standard deviation obtained from the least-square fit. We estimate the stated crystallite size to be accurate within a few nanometers.

The behavior of nc-MgO under quasi-hydrostatic compression is apparently different from that of "bulk" $\mathrm{MgO}$ (Ref. 28) and the difference becomes clearly visible at pressures above roughly $20 \mathrm{GPa}$ in Fig. 2. The decreased incompressibility of nc-MgO in comparison to coarse-grained

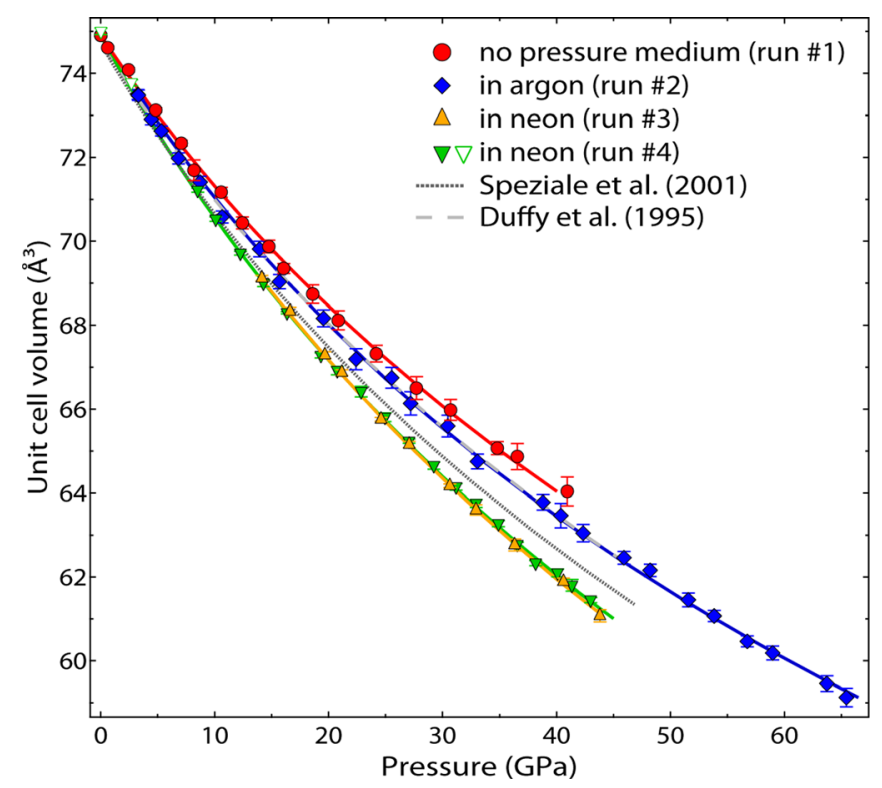

FIG. 2. (Color online) Pressure-dependence of the unit cell volume of nc$\mathrm{MgO}$. Solid curves are third order Birch-Murnaghan equation of state fits to the experimental data. Error bars indicate $1 \sigma$-uncertainties. Open symbols denote data collected during or after decompression. 


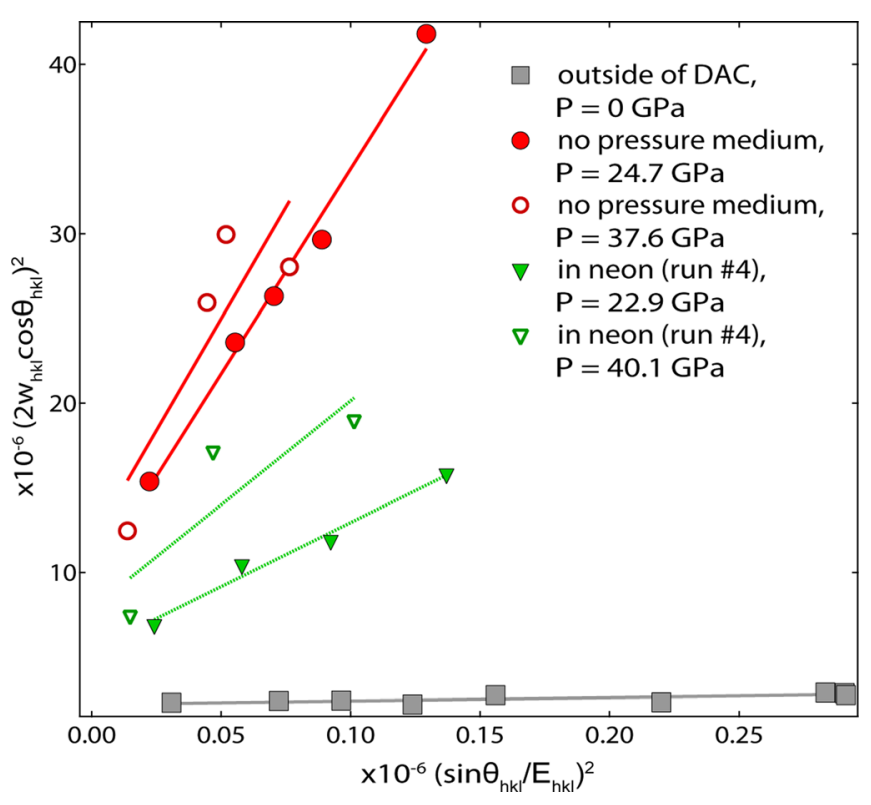

FIG. 3. (Color online) Typical examples of the x-ray line broadening analysis (e.g., Refs.10 and 11). Linear fits of Eq. (1) to the data points at a specific pressure are performed to determine the average crystallite size from the intercept.

$\mathrm{MgO}$ can likely be attributed to the large fraction of atoms within the nc-material that are affected by interfaces between crystallites, which leads to a reduced atomic density and hence a softening of the material (e.g., Refs. 8 and 32). Least-square fits of a third order Birch-Murnaghan equation of state to our data constrain the bulk modulus of nc-MgO to a range between $145.8( \pm 0.1) \mathrm{GPa}$ and $146.7( \pm 0.1) \mathrm{GPa}$ (Table I), which is $8-9 \%$ lower than the bulk modulus of coarse-grained $\mathrm{MgO}$ (160.2 GPa, Ref. 28). For better comparison of $K_{T 0}$ of nc-MgO and $K_{T 0}$ of its "bulk" counterpart, $\left(\partial K_{T} / \partial P\right)_{T 0}$ was fixed to 4 for this analysis. A second fit, where $\left(\partial K_{T} / \partial P\right)_{T 0}$ was not constrained, yielded $K_{T 0}$ values between $150( \pm 0.3) \mathrm{GPa}$ and $152.2( \pm 0.6) \mathrm{GPa}$, with $\left(\partial K_{T} / \partial P\right)_{T 0}$ between $3.47( \pm 0.05)$ and $3.72( \pm 0.03)$. To

TABLE I. Experimental conditions and fitted parameters of the third order Birch-Murnaghan equation of state. $V_{O}$ was always constrained to $74.9 \AA^{3}$ in the least-square fitting. Uncertainties represent $1 \sigma$ - estimated standard deviations (from the fit).

\begin{tabular}{|c|c|c|c|c|c|}
\hline Reference & $\begin{array}{l}\text { Crystallite } \\
\text { size }\end{array}$ & $\begin{array}{l}\text { Pressure } \\
\text { medium }\end{array}$ & $\mathrm{P}_{\max }$ & $\begin{array}{l}K_{T 0} \\
(\mathrm{GPa})\end{array}$ & $\left(\partial K_{T} / \partial P\right)_{T 0}$ \\
\hline This study (run \#1) & $20 \mathrm{~nm}$ & no & $46 \mathrm{GPa}$ & $\begin{array}{l}185.8( \pm 0.4) \\
183.9( \pm 1.2) \\
152.2 \text { (fixed) }\end{array}$ & $\begin{array}{c}4.0 \text { (fixed) } \\
4.18( \pm 0.1) \\
7.53( \pm 0.05)\end{array}$ \\
\hline This study (run \#2) & $20 \mathrm{~nm}$ & argon & $66 \mathrm{GPa}$ & $\begin{array}{l}173.9( \pm 0.2) \\
168.6( \pm 0.8) \\
152.2 \text { (fixed) }\end{array}$ & $\begin{array}{c}4.0 \text { (fixed) } \\
4.3( \pm 0.05) \\
5.32( \pm 0.01)\end{array}$ \\
\hline This study (run \#3) & $20 \mathrm{~nm}$ & neon & $45 \mathrm{GPa}$ & $\begin{array}{l}145.8( \pm 0.1) \\
152.2( \pm 0.6)\end{array}$ & $\begin{array}{c}4.0 \text { (fixed) } \\
3.47( \pm 0.05)\end{array}$ \\
\hline This study (run \#4) & $11 \mathrm{~nm}$ & neon & $42 \mathrm{GPa}$ & $\begin{array}{l}146.7( \pm 0.1) \\
150.0( \pm 0.3)\end{array}$ & $\begin{array}{c}4.0 \text { (fixed) } \\
3.72( \pm 0.03)\end{array}$ \\
\hline Ref. 39 & "bulk" & no & $227 \mathrm{GPa}$ & $177.0( \pm 4.0)$ & $4.0( \pm 0.1)$ \\
\hline Ref. 28 & "bulk" & helium & $52 \mathrm{GPa}$ & $160.2( \pm 0.2)$ & $3.99( \pm 0.01)$ \\
\hline
\end{tabular}

verify these findings, we also analyzed our data by considering each X-ray line individually ("apparent lattice parameters" analysis, e.g., Refs. 33 and 34). For our analysis, we chose the five strongest X-ray reflections (200, 220, 222, 400, 420 ) collected in the second hydrostatic run (run \#4), which we could fit well over the entire experimental pressure range. The calculated bulk moduli range from 146.2 ( \pm 0.4$) \mathrm{GPa}$ to 149.2 ( \pm 0.6$) \mathrm{GPa}$ when fixing $\left(\partial K_{T} / \partial P\right)_{T 0}$ to 4 and from $149.3( \pm 1.0) \mathrm{GPa}$ to $153( \pm 2.8) \mathrm{GPa}$ with $\left(\partial K_{T} / \partial P\right)_{T 0}$ values of 3.52 ( \pm 0.1$)$ to 3.77 ( \pm 0.1 ), see also Table II. In contrast to a previous work on nickel, ${ }^{8}$ we could not detect any clear dependence of $K_{T 0}$ or $\left(\partial K_{T} / \partial P\right)_{T 0}$ on the scattering vector Q.

Our results indicate that the compression behavior strongly depends on the stress conditions in the DAC (Fig. 2 ), consistent with previous studies. ${ }^{35,36}$ Fitting a third order Birch-Murnaghan equation of state (with $\left.\left(\partial K_{T} / \partial P\right)_{T 0}=4\right)$ to our data indicates that the zero pressure bulk modulus increases by about $27 \%$ from $145.8 \mathrm{GPa}$ under hydrostatic conditions (neon as pressure-transmitting medium) to 185.8 GPa under non-hydrostatic conditions (Table I). This value, however, represents an "effective" bulk modulus, i.e., in the plane perpendicular to the experimental compression direction. A pronounced dependence of apparent bulk modulus on the stress conditions in the diamond-anvil cell has also been observed for "bulk" MgO (e.g., Refs. 35 and 36), where the bulk modulus determined from non-hydrostatic compression experiments is $12-13 \%$ larger than the one derived from hydrostatic experiments. Comparison of these results with our data indicates that the effect of deviations from hydrostatic stress condition on the "effective" compression behavior is markedly enhanced in nc-MgO compared to "bulk" $\mathrm{MgO}$. In addition, the spread of derived bulk modulus $K_{T 0}$ based on the "apparent lattice parameters" analysis is significantly larger for the non-hydrostatic experiment compared to the measurements performed in quasi-hydrostatic conditions (Table II). Our general findings on the compressibility of nc$\mathrm{MgO}$ are consistent with a previous comparative study on the hydrostatic compressibility of nc- and "bulk" $\mathrm{Ni}$, where the bulk modulus of nc-Ni $(10 \mathrm{~nm})$ was found to be reduced by about $10 \%$ as compared to coarse-grained $\mathrm{Ni}^{8}$, a finding also supported by computational results. ${ }^{37}$

We could not resolve any difference in the hydrostatic compression behavior between the starting material and the compression behavior of a sample recovered from the nonhydrostatic experiment, which underwent previous crystallite size reduction to about $11 \mathrm{~nm}$ (Fig. 4). This implies that, within our experimental resolution, the bulk modulus does not change between nc-MgO samples with about 20 and $11 \mathrm{~nm}$ starting crystallite size. In contrast to our findings and a previous study on nickel, ${ }^{8}$ no significant difference was found between bulk and nc-3C-SiC under hydrostatic compression conditions. ${ }^{14}$ The crystallite size in the latter study ${ }^{14}$ was about $30 \mathrm{~nm}$. We note that the previous experiments on 3C-SiC (Ref. 14) were limited to pressures below $20 \mathrm{GPa}$, while in our experiments the difference in compression behavior between nc-MgO and its bulk counterpart, becomes clear above roughly $20 \mathrm{GPa}$.

From the x-ray line broadening analysis (Fig. 3), we can derive the change of crystallite size with increasing pressure. 
TABLE II. Fitted parameters of the third order Birch-Murnaghan equation of state using the "apparent lattice parameter" analysis for the hydrostatic loading (run \#4, upper five rows) and the non-hydrostatic experiment (run \#1, lower rows). $V_{0}$ was always fixed to $74.9 \AA^{3}$ in the least-square fitting. The last two columns show the best-fit $\left(\partial K_{T} / \partial P\right)_{T 0}$ when $K_{T 0}$ is fixed to either $150.0 \mathrm{GPa}$ or $152.2 \mathrm{GPa}$, i.e., the values derived for hydrostatic compression (Table I). Uncertainties represent $1 \sigma$-standard deviations (from the fit).

\begin{tabular}{|c|c|c|c|c|c|}
\hline$h k l$ & $K_{T 0}(\mathrm{GPa})$ & $\left(\partial K_{T} / \partial P\right)_{T 0}$ & $\begin{array}{c}K_{T 0}(\mathrm{GPa}) \\
\left(\partial K_{T} / \partial P\right)_{T O}=4\end{array}$ & $\begin{array}{c}\left(\partial K_{T} / \partial P\right)_{T 0} \\
K_{T 0}=150.0 \mathrm{GPa}\end{array}$ & $\begin{array}{c}\left(\partial K_{T} / \partial P\right)_{T 0}, \\
K_{T 0}=152.2 \mathrm{GPa}\end{array}$ \\
\hline 200 & $152.6( \pm 1.5)$ & $3.52( \pm 0.10)$ & $146.2( \pm 0.41)$ & $3.70( \pm 0.02)$ & \\
\hline 220 & $149.3( \pm 1.0)$ & $3.77( \pm 0.07)$ & $146.3( \pm 0.23)$ & $3.72( \pm 0.02)$ & \\
\hline 222 & $147.1( \pm 3.9)$ & $4.14( \pm 0.41)$ & $148.5( \pm 0.93)$ & $3.86( \pm 0.10)$ & \\
\hline 400 & $153.0( \pm 2.8)$ & $3.73( \pm 0.19)$ & $149.2( \pm 0.61)$ & $3.93( \pm 0.04)$ & \\
\hline 420 & $151.8( \pm 2.3)$ & $3.61( \pm 0.16)$ & $146.7( \pm 0.51)$ & $3.74( \pm 0.03)$ & \\
\hline 200 & $173.0( \pm 1.8)$ & $4.65( \pm 0.15)$ & $180.6( \pm 0.6)$ & & $6.66( \pm 0.12)$ \\
\hline 220 & $170.1( \pm 2.3)$ & $5.34( \pm 0.22)$ & $184.9( \pm 1.0)$ & & $7.19( \pm 0.11)$ \\
\hline 222 & $167.3( \pm 6.1)$ & $6.43( \pm 0.66)$ & $192.7( \pm 2.0)$ & & $8.18( \pm 0.16)$ \\
\hline 311 & $197.1( \pm 3.1)$ & $3.76( \pm 0.24)$ & $194.1( \pm 0.8)$ & & $8.03( \pm 0.27)$ \\
\hline 400 & $175.2( \pm 7.1)$ & $4.64( \pm 0.62)$ & $182.6( \pm 1.7)$ & & $6.88( \pm 0.20)$ \\
\hline 420 & $167.3( \pm 5.2)$ & $5.61( \pm 0.51)$ & $184.8( \pm 1.6)$ & & $7.20( \pm 0.15)$ \\
\hline
\end{tabular}

Figure 4 shows the change of crystallite size with pressure in the hydrostatic compression experiments. Even though the data scatter significantly, it appears that the crystallite size remains unchanged upon hydrostatic compression, at least within uncertainties. The data obtained during decompression are not shown, because the crystallite size determination is likely biased by residual stresses in the sample chamber of the DAC.

Upon non-hydrostatic compression, the crystallite size decreases from $19.7( \pm 0.2) \mathrm{nm}$ at ambient pressure to about $7 \mathrm{~nm}$ at high pressures (Fig. 4). Most of the crystallite size reduction takes place upon initial compression (until $10 \mathrm{GPa}$ ), qualitatively consistent with previous studies on nc$\mathrm{MgO} \cdot{ }^{10,12,38} \mathrm{We}$ argue that the observed decrease in apparent crystallite size originates from two distinct features: (1) Actual mechanical reduction of the crystallite sizes and (2)

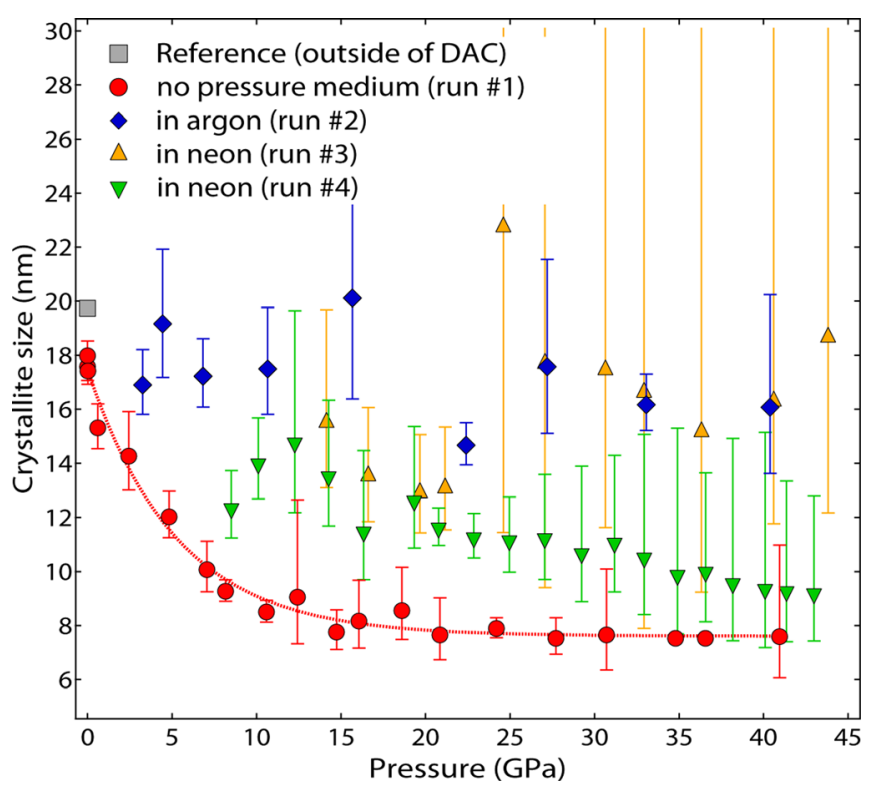

FIG. 4. (Color online) Crystallite size of nc- $\mathrm{MgO}$ as a function of pressure as derived from the analysis of the x-ray line broadening. In the second experimental run performed in neon pressure medium (run \#4), the decompressed sample of the non-hydrostatic run was re-loaded. Error bars indicate $1 \sigma$-uncertainties. The dotted curve is a guide to the eyes. nonhydrostatic stress effects, i.e., crystallites are subdivided into smaller coherently scattering domains upon compression as a result of microstresses (see also Refs. 11 and 13). The first effect results in a permanent decrease of crystallite size, whereas the latter effect is reversible upon decompression. The data collected in run \#4 (where the pre-compressed sample was re-loaded in a hydrostatic pressure medium) indicate an average crystallite size of about $11 \mathrm{~nm}$ (average value over all pressures), implying that most of the crystallite size reduction as determined by $\mathrm{x}$-ray diffraction peak broadening analysis is permanent.

This finding is supported by a previous work, ${ }^{38}$ where high-resolution transmission electron microscopy (HRTEM) was used to derive average crystallite sizes from a decompressed sample of nc-MgO (from above $20 \mathrm{GPa}$ ) to complement in situ determinations of crystallite size using Eq. (1). Excellent agreement was found between the HRTEM measurements on the decompressed sample and the determination from x-ray line broadening analysis at high-pressure, ${ }^{38}$ confirming that the observed crystallite size reduction is mostly permanent.

\section{CONCLUSION}

We find that $\mathrm{nc}-\mathrm{MgO}(20 \mathrm{~nm}$ crystallite size) is more compressible than "bulk" $\mathrm{MgO}$ under quasi-hydrostatic compression. The apparent compressibility of $\mathrm{nc}-\mathrm{MgO}$, however, strongly depends on the experimental stress conditions. Our observations indicate that this dependence is more pronounced for nc- $\mathrm{MgO}$ compared to its coarse-grained counterpart. Finally, we would like to emphasize that the elastic strain derived from x-ray diffraction experiments is mostly related to the crystalline cores; it does not necessarily represent the bulk elastic behavior of a nanocrystalline aggregate. ${ }^{38}$

\section{ACKNOWLEDGMENTS}

The authors thank A. Gleason for providing the nc- $\mathrm{MgO}$ sample. H.M. acknowledges financial support from The German Science Foundation (DFG-SPP1236). This work was 
partially supported by the DFG Grant No. HE 2015/11-1. W.M. acknowledges financial support from the BMBF (Project No. 05K10RFA). TEM sample preparation by A. Schreiber is acknowledged.

${ }^{1}$ H. Gleiter, Prog. Mater. Sci. 33, 223 (1989).

${ }^{2}$ H. Gleiter, Acta Mater. 48, 1 (2000).

${ }^{3}$ H. Dong, D. He, T. S. Duffy, and Y. Zhao, Phys. Rev. B 79, 014105 (2009).

${ }^{4}$ B. Chen, D. Penwell, L. R. Benedetti, R. Jeanloz, and M. B. Kruger, Phys. Rev. B 66, 144101 (2002).

${ }^{5}$ S. Rekhi, S. K. Saxena, R. Ahuja, B. Johansson, and J. Hu, J. Mater. Sci. 36, 4719 (2001).

${ }^{6}$ B. Chen, D. Penwell, and M. B. Kruger, Solid State Commun. 115, 191 (2000).

${ }^{7}$ S. Rekhi, S. K. Saxena, Z. D. Atlas, and J. Hu, Solid State Commun. 117, 33 (2000).

${ }^{8}$ J. Zhang, Y. Zhao, and B. Palosz, Appl. Phys. Lett. 90, 043112 (2007).

${ }^{9}$ J. Xiaogang, Z. Hanzhao, C. Rongzheng, Z. Lei, Z. Qing, L. Jing, and X. Lisong, AIP Conference Proceedings 429, 99 (1998).

${ }^{10}$ A. K. Singh, H. P. Liermann, and S. K. Saxena, Solid State Commun. 132, 795 (2004).

${ }^{11}$ H.-P. Liermann, A. Jain, A. K. Singh, and S. K. Saxena, J. Phys. Chem.; Solids 71, 1088 (2010).

${ }^{12}$ J. Chen, N. Schmidt, J. Chen, L. Wang, D. Weidner, J. Zhang, and Y. Wang, J. Mater. Sci. 40, 5763 (2005).

${ }^{13}$ A. K. Singh, H. P. Liermann, S. Saxena, H. K. Mao, and S. Usah Devi, J. Phys.: Condens. Matter 18, S969 (2006).

${ }^{14}$ H. Liu, J. Hu, J. Shu, D. Häusermann, and H.-k. Mao, Appl. Phys. Lett. 85, 1973 (2004).

${ }^{15}$ B. Chen, D. Penwell, M. B. Kruger, A. F. Yue, and B. Fultz, J. Appl. Phys. 89, 4794 (2001).

${ }^{16}$ F. Birch, J. Geophys. Res. 83, 1257, doi:10.1029/JB083iB03p01257 (1978).

${ }^{17}$ H. k. Mao, R. J. Hemley, and W. L. Mao, in Advances in High Pressure Research in Condensed Matter, edited by S. K. Sikka (NISCOM, New Delhi, 1997), p. 12.
${ }^{18}$ H.-k. Mao, J. Xu, and P. M. Bell, J. Geophys. Res. 91, 4673, doi:10.1029/ JB091iB05p04673 (1986).

${ }^{19}$ H.-k. Mao, P. M. Bell, J. W. Shaner, and D. J. Steinberg, J. Appl. Phys. 49, 3276 (1978).

${ }^{20}$ H. P. Liermann, W. Morgenroth, A. Ehnes, A. Berghäuser, B. Winkler, H. Franz, and E. Weckert, J. Phys.: Conf. Ser. 215, 012029 (2010).

${ }^{21}$ A. P. Hammersley, ESRF International Report No. ESRF97HA02T (1997).

${ }^{22}$ A. K. Singh, J. Phys. Chem. Solids 65, 1589 (2004).

${ }^{23}$ P. Scherrer, Nachr. Ges. Wiss. Göttingen 26, 98 (1918).

${ }^{24}$ J. Langford, J. Appl. Crystallog. 4, 164 (1971).

${ }^{25}$ C. C. Murdock, Phys. Rev. 35, 8 (1930).

${ }^{26}$ M. von Laue, Z. Kristallog. 64, 115 (1926).

${ }^{27}$ B. B. Karki, L. Stixrude, S. J. Clark, M. C. Warren, G. J. Ackland, and J. Crain, Am. Mineralog. 82, 52 (1997).

${ }^{28}$ S. Speziale, C. S. Zha, T. S. Duffy, R. J. Hemley, and H. K. Mao, J. Geophys. Res., B 106, 515, doi:10.1029/2000JB900318 (2001).

${ }^{29}$ W. Qin, T. Nagase, Y. Umakoshi, and J. A. Szpunar, J. Phys.: Condens. Matter 19, 236217 (2007).

${ }^{30}$ J. Sheng, G. Rane, U. Welzel, and E. J. Mittemeijer, Physica E 43, 1155 (2011).

${ }^{31}$ P. P. Chatterjee, S. K. Pabi, and I. Manna, J. Appl Phys. 86, 5912 (1999).

${ }^{32}$ S. Trapp, C. T. Limbach, U. Gonser, S. J. Campbell, and H. Gleiter, Phys. Rev. Lett. 75, 3760 (1995).

${ }^{33}$ B. Palosz, E. Grzanka, S. Gierlotka, S. Stel'makh, R. Pielaszek, U. Bismayer, J. Neuefeind, H. P. Weber, T. Proffen, R. Von Dreele, and W. Palosz, Z. Kristallog. 217, 497 (2002).

${ }^{34}$ B. Palosz, S. Stelmakh, E. Grzanka, S. Gierlotka, and W. Palosz, Z. Kristallog. 222, 580 (2007).

${ }^{35}$ T. S. Duffy, G. Shen, D. L. Heinz, J. Shu, Y. Ma, H.-K. Mao, R. J. Hemley, and A. K. Singh, Phys. Rev. B 60, 15063 (1999).

${ }^{36}$ Y. Fei, Am. Mineralog. 84, 272 (1999).

${ }^{37}$ S.-J. Zhao, K. Albe, and H. Hahn, Scripta Mater. 55, 473 (2006).

${ }^{38}$ H. Marquardt, A. E. Gleason, K. Marquardt, S. Speziale, L. Miyagi, G. Neusser, H. R. Wenk, and R. Jeanloz, Phys.1 Rev. B 84, 064131 (2011).

${ }^{39}$ T. S. Duffy, R. J. Hemley, and H.-k. Mao, Phys. Rev. Lett. 74, 1371 (1995). 